\title{
IDENTIDAD Y DISCRIMINACIÓN EN EL CONTENIDO NO CONCEPTUAL
}

\author{
Justina DÍAZ LEGASPE \\ Universidad Nacional de La Plata \\ CONICET \\ justinadiazlegaspe@yahoo.com.ar
}

RESUMEN: En The Varieties of Reference, Evans sostiene que el contenido perceptual posee una naturaleza no conceptual. Precisamente, los vínculos informacionales entre sujeto y objeto habilitan el pensamiento singular, al permitir la localización del objeto en un entorno egocéntrico. Anclados en algunos casos en estos vínculos, los pensamientos singulares contienen Ideas adecuadas del objeto, dependientes de una determinada clasificación del mismo. Nada en el contenido perceptual equivale a este recorte conceptual del objeto en el pensamiento. Sostendré entonces la necesidad de introducir la idea de una representación no conceptual de cosa que recortaría, de dicho contenido, un punto de anclaje para la representación informacional.

PALABRAS CLAVE: sortales, principio de generalidad, percepción, Evans, protoobjetos

SUmmary: In The Varieties of Reference, Evans claims that perceptual content has a non-conceptual nature. Concretely, the informational links between subject and object allow singular thought by permitting the localization of the object in an egocentric space. Anchored in some cases in these links, singular thoughts contain adequate Ideas of the object that depend on a certain classification of it. Nothing in the perceptual content corresponds to this conceptual cut of the object in thought. I will therefore underline the need to introduce the idea of a non-conceptual representation of thing that will cut, in that content, an anchoring point for the informational representation.

KEY WORDS: sortals, generality constraint, perception, Evans, proto-objets

Desde la publicación de la obra póstuma de Gareth Evans, The Varieties of Reference (1982a), ${ }^{1}$ numerosos autores se han abocado a discutir las características propias del contenido de la percepción; específicamente, si este contenido posee o no una naturaleza puramente no conceptual, independientemente de si se utilizan conceptos para explicarlo o describirlo. ${ }^{2}$ Las pasiones suscitadas por la discusión cobran fuerza debido al hecho de que una noción de contenido perceptual no conceptual, de ser viable, resultaría ser una herramienta importante en la construcción de teorías filosóficas de diversa índole:

${ }^{1}$ En adelante, VR. Véase también, del mismo autor, 1982b.

${ }^{2}$ Agradezco al evaluador anónimo de Crítica por señalarme la necesidad de esta aclaración. 
en filosofía del lenguaje, de la mente, en metafísica y en consideraciones sobre la naturaleza de los conceptos, entre otros.

Cabe trazar aquí una distinción entre contenido perceptual, experiencia perceptual y los juicios o creencias que el sujeto puede tener a partir de la percepción. Evans, en particular, atribuye una naturaleza conceptual a todo el nivel de creencias y pensamientos y, específicamente, a los juicios acerca de la experiencia, mientras que el contenido de la percepción sería completamente no conceptual: entendiéndolo como un sistema que provee de información sobre los objetos al sujeto de pensamiento, Evans considera que éste puede, a lo sumo, controlar ${ }^{3}$ el contenido de los juicios de experiencia o percepción, pero que no es constitutivo de ellos; los juicios acerca de un objeto no incluyen un modo de presentación del mismo como "el objeto responsable de la información recibida". Así, la información adquirida perceptualmente, si bien puede ser representada en forma de una oración abierta, no está en sí misma estructurada por la aplicación de concepto alguno: la aplicación de conceptos se hace exclusivamente en el nivel de la creencia o el pensamiento. El vínculo perceptual entre sujeto y objeto provee al primero, entonces, de información netamente no conceptual sobre el segundo. Por otro lado, contenido perceptual y experiencia tampoco deben ser confundidos: la percepción consiste en un entramado de estados informacionales, y Evans no identifica la experiencia (a la que define como "estado de un sujeto consciente" ( $V R$, p. 157)) con dichos estados. Para el autor, un estado informacional cuenta como experiencia solamente si su contenido no conceptual se halla disponible al sujeto como input o entrada para una creencia o razonamiento conceptual. Así, sólo parece contar como experiencia aquel contenido perceptual que resulte conceptualizable. ${ }^{4}$

De este modo, el pensamiento evansiano, en lo que respecta a la naturaleza conceptual o no conceptual de cada uno de sus componentes, puede dividirse en dos grandes áreas: por un lado, el plano del contenido perceptual, un sistema informacional (esto es, un sistema de habilidades interrelacionadas que nos permiten reunir información acerca del entorno) en el cual no participa concepto alguno; y el plano conceptual del pensamiento o las creencias. Detengámonos en éste por un momento: los juicios o pensamientos singulares se

3 "La información (con contenido $\mathrm{Fx}_{\mathrm{x}}$ ) 'controla' un pensamiento acerca de un objeto particular si y sólo si el sujeto, gracias a la adquisición y conservación de la información, está dispuesto a 'advertir y evaluar' pensamientos sobre el objeto como pensamientos sobre una cosa F” (Sainsbury 1985, p. 125; la traducción es mía).

${ }^{4}$ Para esto, véase McDowell 1996, p. 49. 
estructuran, de acuerdo con Evans, en habilidades diferentes, correspondientes al sujeto y predicado gramatical de las oraciones que podrían expresarlas o, en términos más fregeanos, a sus argumentos y funciones. Así, cada pensamiento o creencia está conformada por una habilidad relacionada con la capacidad de aplicar un concepto, y por una Idea del objeto, la habilidad de pensar en el objeto en cuestión bajo diferentes conceptos. Las Ideas se hallan estrechamente relacionadas con los modos de identificación de los objetos del pensamiento por parte del sujeto: es un conocimiento del objeto por parte del sujeto tal que hace que éste sepa que el pensamiento que está teniendo versa sobre el objeto sobre el que versa y no sobre otro, ${ }^{5}$ y en consecuencia requiere que el sujeto sea capaz de distinguir al objeto por sobre los demás. Para ello, Evans sostiene que todas las Ideas adecuadas de objetos se basan, directa o indirectamente, en la captación, por parte del sujeto, de una propiedad que constituya la base de discriminación fundamental del objeto en cuestión: una propiedad que solamente ese objeto y ningún otro posee, que por tanto lo diferencie de los demás, lo discrimine. Para lograrlo, esta base deberá estar conformada, a su vez, por dos elementos: en primer lugar, la localización espacio-temporal del objeto. En segundo lugar, y en consonancia con esta primera idea, la apelación a una clasificación determinada del objeto bajo un concepto sortal. ${ }^{6}$ Por lo tanto, una propiedad discriminadora tiene por fuerza que ser una propiedad identificatoria: una propiedad que apele a conceptos necesarios para la identificación de los objetos, esto es, a los conceptos sortales adecuados para cada tipo de objeto. ${ }^{7}$ De este modo, las Ideas

5 "Una Idea de un objeto es parte de una concepción de un mundo de objetos de ese tipo, diferenciados unos de otros en ciertas maneras fundamentales. Para todo tipo de objeto, hay una respuesta general a la pregunta “¿Qué hace que sea el caso que haya dos objetos de este tipo en lugar de solamente uno (o dos en lugar de tres)?" " $(V R$, p. 106). Pero no deben confundirse las Ideas con los sentidos fregeanos: independientemente de que alguien las tenga, las Ideas no existen objetivamente. Por otro lado, dos personas con Ideas numéricamente distintas de un mismo objeto pueden, no obstante, captar el mismo sentido (véase la nota 24 en $V R$, p. 104).

${ }^{6}$ Seguimos aquí, al hablar de conceptos sortales, todas las discusiones acerca de cierto tipo de conceptos clasificatorios en la bibliografía sobre el tema. Para una presentación y un desarrollo de estas ideas, véanse Wiggins 2001 y Griffin 1977. En lo sucesivo se hablará de sortales o conceptos sortales. Sin embargo, deberá entenderse que se agrupa bajo este rótulo a los términos de masa sortalizados ("pedazo de arcilla", "pieza de madera"), que poseen las mismas características que los conceptos sortales.

${ }^{7}$ Un número se diferencia de los demás por su posición en una secuencia numérica, y una entidad ficcional se diferencia de las demás por su posición como nodo en una red de características fijadas por un relato en particular. Estas ideas se repiten 
adecuadas de objetos serán relativas a conceptos de sortal o sortaldependientes.

Los conceptos de sortal cumplen esta función de identificación del objeto debido a la falencia de la localización como herramienta para esta tarea. Ahora bien, al contrastar las Ideas de objetos con la información acerca de objetos en que consiste el contenido perceptual, vemos que esa función de identificación se halla ausente en este último. La información permite la localización del objeto, pero nada en este vínculo parece ofrecer un equivalente a la función de identificación que el concepto de sortal cumple en el plano del pensamiento. Puesto que un concepto sortal no podría cumplir este papel en este plano, algo más debería cumplir en él una función no ya de identificación, sino de discriminación, de recorte de una entidad que funcione como término de la relación informacional. En lo que sigue, propondré como candidata para ello a una representación no conceptual de $\cos a$, sosteniendo además que ésta funciona como primitivo o input para la idea, conceptual, de objeto sortalizado.

La sección 1 presentará con mayor detalle estas ideas de Evans acerca del contenido perceptual, Ideas de objeto y el vínculo entre ambas. En ella trataré de mostrar el papel que el concepto de sortal cumple en las Ideas (defendiendo la tesis de que se trata de concepciones sortal-dependientes), y cómo nada cumple ese papel en el plano perceptual, argumentando que el vínculo informacional resulta insuficiente para el recorte del objeto-fuente. La sección 2 insistirá en que el contenido perceptual necesita algo que satisfaga esa función de discriminación, e introducirá la representación de cosa como herramienta para ello. Esta representación deberá enfrentar dos problemas para sustituir adecuadamente a los conceptos de sortal en la percepción de objetos: deberá mostrar que tiene al menos tanta capacidad discriminatoria como éstos, cosa que se probará en la sección 3 , y deberá presentar rasgos que impidan su interpretación como un concepto básico, lo cual será rechazado en la sección 4 . La sección 5 consistirá en una recapitulación de lo dicho que se centrará en la distinción de las funciones de identificación (sortales) y discriminación (representación no conceptual de cosa) que resultan necesarias para percibir y pensar en objetos.

en la concepción de designador canónico de Brandom, donde se diferencian distintos tipos de designadores canónicos para diferentes tipos de entidades (materiales, ficcionales, matemáticas o abstractas). Véase Brandom 1994, segunda parte. 


\section{Evans, la identificación de objetos y el requisito de discriminación}

Interesado tanto en los términos singulares como en los pensamientos singulares que aquellos ayudan a expresar, Evans basa gran parte de las ideas expresadas en $V R$ en el Principio de Russell y la Restricción de Generalidad. Evans retoma el primero ${ }^{8}$ de la obra de Russell (1910), en las siguientes líneas: un sujeto posee un pensamiento con un contenido determinado siempre que le resulte posible concebir cuáles serían las condiciones de verdad para este contenido. ${ }^{9}$ En el caso particular de los pensamientos singulares, parte de este conocimiento está dado por un conocimiento discriminatorio del objeto, un conocimiento de éste como el objeto sobre el cual versa el pensamiento en cuestión. Este "saber cuál" [know-which] está estrechamente relacionado con la Restricción de Generalidad ( $V R$, p. 104); la habilidad a la que Evans denomina "Ideas" de objetos le permite al sujeto pensar en los objetos. Puesto que estas Ideas constituyen los modos bajo los cuales el sujeto piensa el objeto diferenciándolo de otros, esta Idea es la que satisface el requisito de "saber cuál" impuesto por el Principio de Russell. Todas las Ideas adecuadas de objeto contarán entonces con el conocimiento, por parte del hablante, de la existencia de una base fundamental de diferenciación [fundamental ground of difference]. Dependiendo del modo en el cual se relacione el sujeto con dicha base, la Idea será fundamental o no fundamental. Una Idea fundamental será aquella gracias a la cual el individuo discrimine al objeto a través de dicha base de diferenciación y gracias a la cual, por lo tanto, será capaz de valuar directamente, para un objeto determinado, proposiciones del tipo $\langle D \psi$ es $F>$, donde $D \psi$ se entiende como la propiedad en que consiste la base de diferenciación. En for-

\footnotetext{
${ }^{8}$ Existen numerosas versiones del Principio Russelliano. Faria presenta un examen de los principios implicados en su interior que resulta interesante. Véase también la asimilación que realiza McDowell (1990) entre su "requisito de knowwhich" (véase la nota 4) y el Principio Russelliano tal como lo entiende Evans: "El trasfondo esencial para la explicación de Evans de los modos demostrativos de presentación es el principio de que, para tener un pensamiento, uno debe saber en qué consistiría que éste fuera verdadero. Una aplicación de este principio es que si hay un objeto particular que deba ser de cierto modo para que el pensamiento sea verdadero, el sujeto debe saber qué objeto es. Este requisito - el requisito de "saber cuál'- es lo que Evans llama 'el Principio de Russell'” (pp. 256-257; la traducción es mía).

${ }^{9}$ Un sujeto comprende una proposición del tipo $\langle a$ es $F>$ sólo cuando es capaz de comprender oraciones en que la misma propiedad (concepto) se atribuye a otros objetos $(<b$ es $F>$ ), o en las que diferentes propiedades se atribuyen al mismo objeto (Idea adecuada) $(<a$ es $G>$ ).
} 
ma correspondiente, el sujeto dispone de una Idea no fundamental cuando puede realizar esta valuación en forma indirecta, a través de inferencias que incluyen premisas como $<a$ es $F>$ y $\langle a=D>$, donde $a$ parece ser un conocimiento del objeto no relacionado más que de este modo indirecto con la base de diferenciación fundamental.

La base de diferenciación fundamental, entonces, deberá ser capaz de recortar el objeto referente de entre todos los demás objetos posibles. Dada la complejidad del debate acerca de la existencia de propiedades esenciales del objeto, una ruta más sencilla para hallar propiedades que puedan cumplir con este papel es considerar que es la posición del objeto la que asegura su diferenciación respecto de los demás. Así, diferentes tipos de objetos acerca de los cuales podemos pensar que serán diferenciados de cualquier otro objeto por hallarse en un punto particular y no en otro de un determinado entramado: un entramando espacio-temporal para los objetos materiales, un entramado matemático/lógico para los objetos abstractos y un entramado ficcional para los objetos ficticios. Incluso en el caso de objetos materiales que no percibimos directamente o que no podríamos percibir, basta suponer su existencia y su naturaleza material para dar por sentado que existe una ubicación determinada para ellos en el espacio-tiempo. Por tanto, un componente fundamental de la base de diferenciación es la capacidad de localizar al objeto en un determinado entramado.

Sin embargo, este componente se basa en el supuesto de que dos objetos no pueden ocupar al mismo tiempo una misma coordenada en el entramado en cuestión. Y si bien esto es cierto en la mayoría de los casos, existe la posibilidad de que dos objetos ocupen exactamente el mismo lugar. En palabras de Evans:

Es tentador decir, de los objetos que existen en el espacio, que lo que diferencia a unos de otros en un determinado momento es que ocupan una posición espacial particular en ese momento. Pero esto no es del todo correcto, dado que aunque dos Gs pueden no ser capaces de compartir una posición en un tiempo determinado, un G puede ser capaz de compartir una posición con una cosa de tipo diferente: por ejemplo, una estatua y un pedazo de arcilla. La respuesta a la pregunta de qué diferencia una estatua de todas las demás cosas en un momento dado se da al citar (i) la posición que ocupa en ese momento y (ii) el hecho de que es una estatua. (VR, p. 107; las cursivas y la traducción son mías.)

Es por esto que la localización, por sí sola, se muestra insuficiente para cumplir el papel de diferenciadora que Evans le pide a la base 
fundamental que da asiento a las Ideas adecuadas de objeto. La localización del objeto no basta para satisfacer esta tarea: el sujeto no sólo debe saber que el objeto está ahí, sino que necesita saber qué tipo de cosa es ésa que está ahí; necesita saber que hay un $F$ ahí. Para completar este requisito de discriminación, Evans opta por recurrir a la idea de identificación, esto es, a la idea de que podemos discriminar un objeto de cualquier otro objeto en el mundo cuando sabemos no sólo dónde se halla, sino principalmente de qué tipo de objeto se trata. Así, la base fundamental de diferenciación consistirá en la identificación del objeto como un objeto de cierto tipo, y sólo secundariamente como un objeto en un lugar determinado. Ahora bien, desde el debate suscitado por las ideas de Geach (1962 y 1967) la noción de identidad se ha relacionado con los llamados conceptos de sortal y términos de masa; en pocas palabras, conceptos que pueden dar respuesta a la pregunta sobre el qué es de la cosa. Son estos conceptos los que cumplen con este requisito de identificación en la base fundamental de diferenciación. La inclusión de conceptos sortales en esta base es aún más fuerte: no se trata solamente de que la identificación que sólo estos conceptos pueden proporcionar ${ }^{10}$ deba acompañar al requisito de localización del objeto, como si se tratara de dos componentes diferentes e independientes. Se trata, en concreto, de que el concepto de sortal en cuestión determinará, de entrada, el tipo de localización que se busque (si se clasifica al objeto como un número, se buscará localizarlo en un entramado matemático y no espacio-temporal, por ejemplo). Así, el requisito de discriminación que requieren las Ideas adecuadas se satisface a través de la identificación que sólo la aplicación de un concepto sortal puede realizar, determinando tanto el tipo de localización deseada como el tipo de objeto que se busca localizar. La base fundamental de identificación resulta ser sortal-dependiente y, por lo tanto, resulta ser no sólo diferenciadora sino, además, identificatoria.

El estudioso de Evans puede hacer aquí un alto: ${ }^{11}$ solamente en la posesión de Ideas fundamentales tiene el sujeto un conocimiento

${ }^{10}$ Los demás conceptos que podrían aplicarse a un objeto no logran identificarlo: sólo los conceptos de sortal pueden hacerlo; basta pensar en la capacidad de identificación de un objeto que poseen conceptos clasificatorios no sortales (como rojo) o conceptos que no llegan a ser sortales sino meros "testaferros", como el concepto de "objeto". Véase, por ejemplo, Griffin 1977 sobre esto. Por otro lado, es notable la influencia de Strawson en este punto: es él quien diferencia, en Strawxon 1959, entre conceptos clasificatorios y conceptos atributivos, esto es, entre conceptos sortales y no sortales.

${ }^{11}$ Agradezco al evaluador anónimo de Crítica por señalarme este punto. 
directo de la base fundamental de diferenciación y, en consecuencia, la necesidad de aplicar un sortal al objeto de sus pensamientos. Pero en las Ideas adecuadas y en particular en las Ideas no fundamentales, el sortal parece volverse innecesario. Así como es posible tener una Idea no fundamental del objeto cuando se desconoce su ubicación pero se supone que es posible localizarlo en el espacio, ${ }^{12}$ es posible también tener una Idea no fundamental cuando el sujeto (a) desconoce a qué tipo de cosa pertenece el objeto localizado y (b) clasifica el objeto bajo un sortal equivocado. ${ }^{13}$ A este respecto, Evans señala:

$[\mathrm{N}$ ]o parece ser verdadero que las identificaciones demostrativas deban acompañarse de un sortal que determine los límites de la cosa en el espacio y el tiempo. He permitido (4.3) que una Idea fundamental de objeto implique tal sortal, pero una identificación demostrativa no constituye en sí misma, necesariamente, una Idea fundamental. Será adecuada sin ser fundamental en tanto el sujeto conozca lo que hace que una proposición de identidad de la forma [Esto $=$ el $\mathrm{G}$ en $\Pi, t]$ sea verdadera, y el sujeto puede saber lo que hace verdaderas tales proposiciones sin saber, de hecho, el tipo de cosa de que se trata, siempre y cuando exista la posibilidad de descubrir el tipo de cosa, y sepa cómo hacerlo. ( $V R$, p. 178)

Así, el sujeto puede tener Ideas estrechamente relacionadas con los vínculos informacionales proporcionados por la percepción, sin que esto requiera necesariamente la aplicación de un concepto de sortal al objeto en la Idea correspondiente. Sólo las Ideas fundamentales requieren que el sujeto conozca la base fundamental de diferenciación directamente: en los casos de Ideas adecuadas pero no fundamentales, el sujeto deberá ser capaz de suponer la existencia de esa base aun cuando no la conozca. Aparentemente, esto permite relajar el requisito de identidad: tal y como señala Evans, en estos casos no es necesario que el sujeto sepa a qué tipo pertenece el objeto. Basta que suponga que existe ese tipo y que sea capaz de averiguarlo de ser necesario.

A modo de respuesta puede ensayarse lo siguiente: la no fundamentalidad de las Ideas adecuadas y el consecuente relajamiento del requisito de identificación no necesariamente elimina la dependencia

${ }^{12}$ Esto contiene las dos posibilidades señaladas por el evaluador: tanto la posibilidad de que el sujeto no sea capaz de ubicar el objeto en su entorno egocéntrico, como la posibilidad de que sea capaz de hacerlo pero no logre trazar una conexión entre este punto egocéntrico y otro punto en el espacio objetivo.

${ }^{13}$ Como sucede en los casos tipo Donnellan; VR, sección 6. 
de sortal; solamente la atenúa. Efectivamente, lo que el sujeto conoce es que existe un juicio de identidad entre el objeto identificado bajo un modo de presentación demostrativo ("esto") y el mismo objeto determinado por la posesión de una base fundamental de diferenciación. Sin embargo, aun cuando se desconozca el sortal correspondiente a dicha base - y, por lo tanto, a dicho objeto-, el lugar de sortal será ocupado, en tanto no se haya realizado el descubrimiento mencionado por Evans, por un testaferro de sortal, un concepto insuficiente para la identificación precisa pero que ocupa el lugar que ocuparía el sortal adecuado cuando se carece de éste. ${ }^{14}$ Del mismo modo, el desconocimiento de la localización exacta del objeto tampoco implica la desaparición del requisito de localización; simplemente se reemplazan temporalmente las coordenadas del objeto por conceptos que cubran dicho lugar ("en alguna parte", por ejemplo). Así, en casos como (a), el sujeto piensa en un objeto en calidad de "esto", dando por sentado que es verdadero que [esto $=\mathrm{el} \mathrm{G}$ en $\Pi, t]$, donde $\Pi$ y $t$ corresponden a un lugar y tiempo conocidos, y $G$ es un testaferro de sortal que podría ser reemplazado, con el descubrimiento eventual, por el sortal correspondiente. Así, incluso las Ideas no fundamentales son, aunque no sea más que indirectamente, sortal-dependientes, incluso ante el desconocimiento del sortal en cuestión.

Muchas de las Ideas de objetos que poseemos están basadas en la información que recibimos a través de los sentidos. En estos casos, existe un nexo directo entre la Idea en cuestión y el vínculo informacional que controla dicha Idea, aun cuando dicho nexo no debe entenderse como constitutivo del contenido de la Idea. Es precisamente el vínculo el que posibilita la localización del objeto. La información obtenida por éste se articula, en primera instancia, en el contexto del espacio egocéntrico ${ }^{15}$ del sujeto (y sólo en un segundo momento lógico se establece una conexión entre dicho espacio y el mapa objetivo del lugar en el que el sujeto se encuentra): las coordenadas espacio-temporales en que se ubican los objetos materiales se hallan estrechamente relacionados con las posibilidades de movimiento del cuerpo del mismo y las acciones posibles hacia ellos. Por eso, este

\footnotetext{
${ }^{14}$ Véase Griffin 1977. El concepto de "objeto" es un clásico ejemplo de testaferro de sortal.

${ }^{15}$ Es en este punto donde se reconoce más claramente la influencia de Strawson 1959 en Evans. La localización es siempre, primeramente, egocéntrica, y sólo después objetiva, para permitir a un individuo que no sabe dónde está (en qué lugar objetivo) tener pensamientos que contengan modos de presentaciones demostrativas del objeto. Sin embargo, ambos espacios son el mismo espacio, individualizado de dos modos diferentes.
} 
vínculo cumple con una función localizante. Sin embargo, los datos que un vínculo informacional perceptual nos proporciona no dejan de ser lo que tradicionalmente se conoce como datos de los sentidos: una masa desarticulada de diferentes propiedades perceptuales o qualias repartidos en un escenario perceptual. Es necesario, entonces, dotar de cierta estructura a dicha masa, recortarla en objetos. Así como en el nivel conceptual algo necesita cumplir el papel identificatorio que la localización efectiva requiere, en el nivel perceptual es necesario, también, un paso previo: el recorte de la entidad que funcionará como anclaje del vínculo informacional.

En su reseña del libro de Evans, Sainsbury (1985) introduce una distinción entre el objeto-meta [target] (aquí, objeto-fuente), entendido como aquel del cual deriva la información sobre la que se basa el pensamiento acerca del objeto, el objeto vinculado informacionalmente con el sujeto, y el objeto en cuanto objeto de pensamiento, el Objeto. Para que pueda ser atribuido al sujeto un genuino pensamiento singular - en casos de pensamientos basados en la información-, ambas entidades deben coincidir, aunque se admite la posibilidad de errores. Como hemos visto, la Idea fundamental se relaciona estrechamente con el Objeto; es una representación de la entidad sobre la que se piensa que se halla previamente conceptualizada o bajo la aplicación de conceptos de sortal, directa o indirectamente. Sin embargo, al considerar el objeto-fuente nos hallamos ante la necesidad de recortar de algún modo aquello a lo cual se aplicará, durante la conceptualización, el concepto sortal correspondiente. En otras palabras, para que pueda haber identificación del objeto en el nivel conceptual debe haber un recorte previo del mismo en el nivel no conceptual o perceptual; la percepción misma debe proporcionar elementos que muestren una mayor estructuración que los meros datos sensoriales, de modo tal que el sujeto pueda enfocar su atención en ellos y clasificarlos, en el nivel conceptual, bajo sortales. Al igual que los conceptos sortales en el nivel conceptual, sea lo que sea que cumpla con esta función en el nivel perceptual no conceptual, debe poseer capacidad discriminatoria, de manera tal que el sujeto, ya en su percepción, sea capaz de percibir segmentos de su entorno perceptual qua objetos, sea capaz de rastrearlos y de reconocerlos si se presentan nuevamente, anclando en ellos el vínculo informacional. Y sea lo que sea que cumpla con esta función en el nivel perceptual, no debe ser de naturaleza conceptual: apelar al uso de conceptos (sortales o de cualquier tipo) en el nivel de la percepción eliminaría la naturaleza puramente no conceptual de la información proporcionada por la percepción. La información localizante del objeto en el espacio egocéntrico no basta 
para satisfacer esta tarea: el sujeto no sólo debe saber que el objeto está ahí, sino que necesita saber que hay algo ahí, aun cuando en este nivel no conceptual no pueda hablarse todavía de qué tipo de cosa se trate este algo.

La identificación sólo puede ser realizada por conceptos de sortal. Por lo tanto se vuelve necesario reemplazar, en el nivel perceptual, la idea de identificación por un correlato que pueda practicarse en el nivel no conceptual y que, al igual que la localización, se base en habilidades del sujeto y no en la aplicación de conceptos. Estas habilidades deberán permitir al sujeto recortar al objeto y diferenciarlo no sólo de otros objetos radicalmente diferentes, sino de objetos del mismo tipo; por otro lado, la discriminación del objeto debe ser lo suficientemente fina como para poder dar cuenta de la presencia de dos entidades de diferente tipo en las mismas coordenadas espaciotemporales.

Queda claro que no es una opción viable considerar, como lo harían los seguidores de la idea de la conceptualización como una "segunda naturaleza" humana, ${ }^{16}$ que la percepción ya implica la aplicación de conceptos sortales. Evans incorpora sortales a las Ideas de objeto puesto que sólo estos conceptos proporcionan una respuesta a la pregunta aristotélica por el qué-es de la cosa y, por lo tanto, proporcionan los criterios de individuación y persistencia ${ }^{17}$ adecuados para la delimitación de los objetos que anclan la localización. Si trasladamos la discriminación de la que son capaces los conceptos de sortal al plano de la información proporcionada por la percepción se obtienen consecuencias no del todo deseables: por ejemplo, en casos de ignorancia de sortal, como hemos visto, el sujeto no podría determinar los límites o condiciones de identidad del objeto ante el cual se halla (recordemos que los testaferros de sortal son insuficientes, por sí solos, para este fin), y entonces no podría determinar si basta tener sus coordenadas de localización para discriminarlo de otros objetos. ${ }^{18}$ Por otro lado, de trasladar los conceptos clasificatorios a la información perceptual se perdería, como es evidente, la

${ }^{16}$ McDowell 1996 es un ejemplo de este tipo de posturas.

${ }^{17}$ Véase Griffin 1977 para un examen de los criterios de individuación asociados a los conceptos de sortal y masa.

${ }^{18}$ Sin el concepto de estatua o arcilla, el sujeto no contaría con el criterio adecuado para saber que un pedazo de arcilla y una estatua son dos objetos que ocupan el mismo lugar en un determinado momento, y, sobre la base de la sola localización, se vería obligado a sostener que se trata de la misma cosa, no pudiendo explicar después por qué el objeto sigue siendo el mismo ante el reemplazo de una de sus partes. Sobre el argumento del reemplazo, véase Thomson 1998. 
naturaleza no conceptual de la misma que Evans desea preservar. Por consiguiente, se hace evidente que otra cosa diferente de un concepto tiene que desempeñar, en el plano informacional, el papel discriminatorio que los conceptos tienen en el plano conceptual y que no puede ser satisfecho solamente por la capacidad de localización del objeto-fuente.

\section{Protoproposiciones, puntos y cosas}

Si las cosas son así, algo en el contenido perceptual debe cumplir el papel discriminatorio que los conceptos sortales cumplen en el nivel conceptual. Por lo tanto, debemos examinar con cuidado el requisito de identificación que Evans atribuye a las Ideas de los objetos. Evans satisface este requisito apelando a la sortalización de los Objetos, a la dependencia de sortal de las Ideas adecuadas: sólo la capacidad de identificar al objeto como un objeto de cierto tipo permite al individuo diferenciarlo de otros objetos de la misma clase o localizados en las mismas coordenadas espacio-temporales. Esto se debe a que la aplicación de conceptos de sortal proporciona criterios de identidad y persistencia que nos informan sobre los límites espacio-temporales y posibilidades de cambio de los objetos pertenecientes a cada tipo, que determinan a su vez el tipo de localización que debemos suministrar en la base fundamental de diferenciación. A fin de hallar un correlato de esta función en el nivel de la información perceptual, el criterio de discriminación puede ser interpretado de otros modos, sin que sea estrictamente necesario que el sujeto apele a conceptos que le permitan identificar al objeto que percibe. Recordemos que lo que este requisito exige es la posibilidad de diferenciar al objeto de cualquier otro, sea del tipo que sea y esté en las coordenadas en que esté. Menos que esto en realidad, puesto que basta con que pueda diferenciarlo de los demás objetos presentes en su entorno perceptual, objetos que pueden ser del mismo tipo (esto es, muy similares en su forma al objeto en cuestión), o situados en las mismas coordenadas espacio-temporales (esto es, objetos que no sólo comparten su forma - o parte de ella-, sino también su localización). Ambas tareas, sostendremos, pueden ser realizadas sin necesidad de apelar a conceptos sortales si contamos con un equivalente de éstos en el plano no conceptual. De este modo, el individuo podría establecer que ahí hay una entidad (digamos, una cosa), sin necesidad de determinar en este nivel de qué tipo de objeto se trata. Se trata entonces de dar con un tipo de satisfacción diferente del requisito de discriminación. 
¿Qué equivalente no conceptual del concepto de Objeto sortalizado puede cumplir el papel discriminatorio que necesitamos? Sostendremos que una representación (no conceptual) de cosa puede desempeñar este papel. ${ }^{19}$ Supongamos, como supone Peacocke (1992), ${ }^{20}$ que el contenido representacional de las percepciones o la información proporcionada por la percepción puede dividirse en dos niveles: un primer nivel más básico consistente en un tipo espacial, llamado escenario: un modo de "rellenar", por así decirlo, el espacio alrededor del sujeto especificado por un origen, el cuerpo del sujeto, y ejes articulados egocéntricamente en relación con aquel: arriba/abajo, izquierda/derecha, delante/detrás; y un segundo nivel no identificable con el escenario pero tampoco con el contenido conceptual de un juicio perceptual, el nivel de las protoproposiciones. Ahora bien, en el escenario el individuo percibe puntos identificables en relación con el origen y los ejes, de modo que para cada punto, cabe determinar si existe en él una superficie con determinadas propiedades (textura, brillo, temperatura, grado de solidez y, en el caso de recibir información visual, dirección, intensidad y carácter de la fuente de luz que lo ilumina); así, cada punto está cualitativamente cargado, como una suerte de conjunción de qualias de diverso género. A diferencia de éste, el nivel protoproposicional ${ }^{21}$ no parece fragmentar la infor-

${ }^{19}$ En lo que sigue, usaremos "representación" para dar cuenta de una categoría aplicable en la percepción pero que, por estar vinculada a la acción y no a la intelección, no puede considerarse como concepto. Subyace a esto, por supuesto, un presupuesto sobre la naturaleza de los conceptos que los liga a los contenidos y actitudes proposicionales. Para más claridad, es posible entender la idea de "representación de cosa" en términos de modelos o representaciones perceptuales que construyen, a partir de perceptos de colores, formas y volúmenes, representaciones tridimensionales de objetos cerrados y autocontenidos. Se puede seguir en este punto a Marr y sus modelos de representaciones en el nivel de 2.5 para llegar a representaciones de objetos tridimensionales, tal y como lo sugiere el evaluador anónimo, a quien le agradezco su observación. Por otro lado, es necesario aclarar en este punto que no se considera que la información perceptual (y, por lo tanto, las representaciones de $\cos a$ operativas en ella) puedan formar parte de actitudes proposicionales: no es la percepción misma, lo percibido, lo que entra en reportes de percepción ("Juan vio a María en la cocina"), sino proposiciones (conceptualizadas) de aquello que es informado por la percepción.

${ }^{20}$ Si bien tanto Peacocke como Evans tratan el contenido perceptual como no conceptual y basado en la capacidad del sujeto perceptor de localizar objetos en su entorno egocéntrico, existen diferencias importantes entre ambos planteamientos; véase McDowell 1990 y Peacocke 1991.

21 "Una protoproposición contiene un individuo o individuos junto con una propiedad o relación. Cuando una protoproposición es parte del contenido representacional de una experiencia, la experiencia representa la propiedad de relación en la protoproposición como si se dieran entre el individuo o individuos que también contiene. 
mación proporcionada por la percepción del entorno en puntos sino en totalidades o entidades con propiedades y relaciones. ${ }^{22}$ Al hablar de los constituyentes de las protoproposiciones, Peacocke habla de objetos, sin problematizar el paso de los puntos en los escenarios a este tipo de entidades. Así, las protoproposiciones están conformadas por objetos (no conceptualizados siquiera bajo el concepto de objeto) que instancian propiedades y relaciones, en tanto que las proposiciones o los pensamientos están conformados o constituidos por conceptos (incluyendo al concepto de objeto), en un esquema similar al planteado por Evans en su diferenciación de un nivel conceptual y uno no conceptual. El hecho de que un contenido representacional pueda estar constituido por objetos sólo puede entenderse cuando se considera que, en este nivel, las protoproposiciones son un recorte de aquello que se percibe pero aún no se conceptualiza: de este modo, la protoproposición [esa cosa es roja $]^{23}$ no está conformada por los conceptos correspondientes, sino que debe entenderse - a mi juicio - como un recorte del entorno realizado por el acto de prestar más atención a ciertos segmentos y aspectos del entorno perceptual o escenario que a otros, de modo que sus componentes son realmente los objetos reales tal y como los percibe el sujeto, con sus propiedades y relaciones. De acuerdo con Peacocke, las protoproposiciones cumplen un papel importante en el almacenaje y la recuperación de información sobre las entidades en el entorno: dada la riqueza de detalles de la información perceptual analógica (Dretske 1981) propia del escenario, el almacenaje de todos los datos es imposible, y por ello, el individuo almacena sólo fragmentos de la información perceptual (las protoproposiciones) que resultan relevantes o a los que se presta atención. Así, el conjunto de las protoproposiciones está

Escribo sobre protoproposiciones en lugar de sobre protopensamientos porque las protoproposiciones contienen objetos, propiedades y relaciones y no los conceptos relacionados con ellos. Escribo sobre protoproposiciones, porque, de acuerdo con esta explicación, no se determinan como parte del contenido de una experiencia fijada por algún contenido conceptual que la experiencia posea" (Peacocke. 1992, pp. 118-119; la traducción es mía).

${ }^{22}$ Estas totalidades o entidades - lo que en términos de Peacocke puede llamarse "protoobjetos" y en los míos "cosas" - a las que se atribuyen propiedades y relaciones no son, desde ya, cualquier totalidad, así como no se trata de cualquier relación ni cualquier propiedad: cabe suponerse que son propiedades y relaciones perceptuales muy simples, probablemente relacionadas con lo perceptual (Peacocke cita "ser cuadrado", "curvo", "paralelo a", etc.). Lo mismo debe suceder con los protoobjetos.

${ }^{23}$ El uso de los corchetes cuadrados señala que se trata de protoproposiciones y, por consiguiente, que su contenido no es conceptual. 
conformado por recortes de información, por así decirlo, del escenario perceptual, aptos para ser almacenados. Este almacenaje permite además la reidentificación de los protocomponentes aun cuando sean percibidos desde otro ángulo o en otro contexto.

Sin embargo, esto no explica todavía el paso de los puntos de los escenarios a los protoobjetos, esto es, a los objetos reales no conceptualizados tal y como son contenidos en las protoproposiciones. Peacocke no se interesa por este salto. Pese a ello, es posible abandonar el aspecto ontológico y concentrarnos en la mente del sujeto perceptor para advertir que, al prestar atención a cierto segmento de su entorno, está constituyendo algunos fragmentos del mismo en unidades que anclan su percepción, que llaman su atención. ${ }^{24}$ Estos conjuntos de fragmentos son las cosas que el sujeto percibe, los objetos tal y como aparecen en su experiencia perceptual aun cuando no ha aplicado concepto alguno ni ha abstraído todos ellos. Así, si bien desde el nivel de los escenarios el sujeto percibe puntos, en el nivel superior de la información perceptual el sujeto percibe protoobjetos. Estos protoobjetos no corresponden aún a los objetos percibidos en el nivel conceptual (mesas, sillas, tazas), ${ }^{25}$ que requieren la clasificación de las entidades bajo sortales. Quiero establecer una identidad entre esta noción de protoobjeto y mi representación no conceptual de "cosa", en oposición a la noción conceptual de objeto. ${ }^{26}$ Es posible, entonces, establecer una diferencia entre la percepción no conceptual de cosas y el reporte de percepción, en el nivel conceptual, que incluye la referencia a objetos sortalizados; los seres humanos, en tanto seres conceptuales, reportan la información perceptual obtenida de su entorno refiriéndose a entidades como sillas, mesas y tazas, esto es, por objetos. En cambio, en el nivel de la percepción la información recibida del entorno no cuenta con la presencia de objetos sortaliza-

${ }^{24}$ La razón por la cual sólo algunos segmentos de su campo perceptual atraen la atención del sujeto y lo conducen a constituir objetos o cosas a partir de los mismos responde a factores relacionados con propiedades cualitativas de dicho campo (contraste entre formas y colores que se percibe como forma y fondo, por ejemplo) que son mencionadas más abajo. Véase, para esto, Hirsch 1982.

${ }^{25}$ Esto, independientemente de las ideas que Peacocke pudiera tener al respecto: si se trata de modos de presentación puramente no conceptuales de las entidades en el entorno, no deberían ser sortalizados, sino meramente percibidos como totalidades en las que inhieren propiedades y relaciones observacionales simples.

${ }^{26}$ Si bien "objeto" no es un término ni de sortal ni de masa, sino un testaferro de sortal (Griffin 1977), puede argumentarse que sigue siendo un concepto. Más adelante defenderé que la noción de cosa no es un concepto de ningún tipo; esto no excluye que exista un concepto correspondiente a la noción no conceptual de cosa, un concepto del tipo de objeto. 
dos: el objeto fuente de la información perceptual es una cosa, una entidad que aún no ha sido clasificada bajo un concepto. El mundo de la percepción es un mundo de cosas, mientras que el mundo del pensamiento es un mundo de objetos.

Ahora bien, la mera percepción de puntos cualitativamente diferentes no asegura la percepción de cosas. Debe ser posible, además, determinar cuándo una colección de puntos conforman una cosa delimitada. Para ello podemos echar mano del concepto de articulación de objetos que presenta Hirsch $(1982)^{27}$ al tratar el problema de la identidad de objetos en casos de ignorancia de sortal; puesto que en estos casos no hay criterios de persistencia y demarcación para el objeto, algo será considerado qua objeto (en nuestro vocabulario y en este nivel, qua cosa) sólo si es considerado

(i) una cosa articulada, y

(ii) una cosa persistente.

La articulación se vincula con la posibilidad de diferenciar la entidad como un todo que se recorta contra un fondo (tal como se señala en la psicología de la Gestalt), mientras que la persistencia está vinculada a la continuidad de las propiedades principales del objeto en el espacio y en el tiempo. Un conjunto o colección de puntos serán entonces considerados una cosa articulada cuando la totalidad resultante de su consideración conjunta presente ciertas propiedades ${ }^{28}$ emergentes o supervenientes de las propiedades de cada uno de los puntos individuales: dichos puntos deberán presentar un alto grado de homogeneidad entre sus propiedades de modo tal que éstas los diferencien de los puntos inmediatamente contiguos, haciendo posible su percepción conjunta como una entidad recortada contra el fondo y claramente delimitada. Sumado a esto, los movimientos y manipulaciones del sujeto alrededor y sobre dichos puntos confirmarán que dichas cualidades siguen vinculadas por un lazo de homogeneidad en formas previsibles (que el cambio de perspectiva produce los cambios

\footnotetext{
${ }^{27}$ Cabe aclarar que Hirsch no se vale de esta regla en un nivel preconceptual, sino que se usa solamente en el nivel conceptual cuando no se poseen sortales. En este trabajo hago uso de las condiciones para la percepción de algo como un objeto articulado y persistente en el nivel preconceptual, alejándome de Hirsch

${ }^{28}$ Esto es: (a) contraste de límites entre el entorno y el objeto; (b) homogeneidad cualitativa del objeto; (c) movilidad separada; (d) cohesión dinámica; (e) regularidad de forma y (f) formación de articulaciones y ausencia de separación de límite. Así, una porción de materia continua constituye un objeto unificado estándar si y sólo si tiene un grado relativamente alto de articulación.
} 
esperados, digamos) y que el objeto posee una movilidad separada de otros puntos contiguos. Los requisitos para la consideración de la cosa como persistente exigen que ésta:

(1) sea espacio-temporalmente continua;

(2) sea cualitativamente continua y

(3) minimice los cambios de sus propiedades (exceptuando el mero cambio de lugar realizado por otro o activo en caso de organismos vivos).

Esto significa que todo cambio cualitativo o espacio-temporal sufrido por el objeto deberá ser gradual, esto es, que la alteración de sus propiedades de un momento a otro o al moverse o ser movido de un lugar a otro serán casi imperceptibles para el sujeto. ${ }^{29} \mathrm{Si}$ esto es así, un sujeto considerará una serie de puntos como una cosa cuando satisfagan todos estos requisitos, una cosa capaz de componer una protoproposición. Por supuesto, esto no es el producto de una inferencia conceptual; no sería del todo correcto, tampoco, considerar esto como el producto de una inferencia no conceptual. Esta consideración de la presencia de cosas ante ciertos rasgos percibidos en el entorno perceptual es inmediata cronológica y lógicamente, aunque para comprenderla resulte de utilidad fragmentar lo que en la mente del sujeto perceptor sucede en un paso en una secuencia de pequeños pasos con el aspecto de una inferencia.

Si contamos con una representación no conceptual de cosa, ésta puede satisfacer el requisito de discriminación implicado en las Ideas adecuadas. De la misma manera en que el concepto de sortal determina el contenido de la base fundamental de diferenciación, la representación no conceptual de cosa proporciona un anclaje, un objeto-fuente para la relación informacional entre sujeto y objeto que permite, a su vez, la localización de éste y el control de esta información sobre los pensamientos singulares acerca del objeto en cuestión. Veamos entonces cómo esta noción de cosa puede satisfacer el requisito de discriminación.

\section{Cosas y discriminación}

Para que sea posible esta correlación de la identidad conceptual por medio de sortales con la discriminación por medio de la distinción

${ }^{29}$ Hirsch mismo advierte la vaguedad presente en la determinación de cuándo un cambio deja de ser gradual para ser abrupto. No creo que sea posible encontrar una solución no casuística y debidamente general para resolver este problema. 
de cosas, el contenido perceptual deberá estar estructurado tanto por vínculos informacionales como por el recorte del entorno en cosas articuladas y persistentes; la discriminación que permiten los conceptos clasificatorios en el nivel conceptual será realizada por la representación de cosa en el nivel no conceptual. Sin embargo, sostener esta idea implica atravesar con éxito dos escollos: en primer lugar, demostrar que la representación de cosa permite una discriminación al menos tan fina como la que permitiría un concepto de sortal; ambas herramientas teóricas deben demostrar ser por lo menos igual de eficaces, cada una en su ámbito de aplicación. Por otro lado, es necesario responder a la posible objeción de que la noción de cosa sólo puede ser entendida como un concepto muy básico. En esta sección abordaremos el primer problema, dejando el segundo para la siguiente.

Para que la representación de cosa satisfaga adecuadamente el requisito de discriminación, debe permitirnos diferenciar: (1) un objeto de tipo $G$ de un objeto de tipo $F$, situados ambos en diferentes coordenadas espaciales pero en el mismo tiempo; (2) un objeto de tipo $\mathrm{G}$ de otro objeto de tipo $\mathrm{G}$ (situados en diferentes coordenadas espaciales y temporales); y (3) un objeto $\mathrm{G}$ de otro objeto $\mathrm{F}$ (situados ambos en las mismas coordenadas espacio-temporales). Si logra hacer esto sin apelar a la identidad de los objetos (a su pertenencia a los tipos $\mathrm{G}$ y $\mathrm{F}$, esto es, sin aplicar sortales a los objetos en cuestión), demostrará tener la capacidad de discriminación necesaria para satisfacer adecuadamente este requisito.

(1) En primer lugar, la representación de cosa permite diferenciar dos objetos pertenecientes a diferentes tipos siempre que se considere que la diferenciación no se realiza (ni en éste ni en ninguno de los casos subsiguientes) de modo abstracto o general: puesto que no se discute la naturaleza de la identidad de las entidades sino la capacidad epistémica del sujeto de percibirlos como cosas diferenciables entre sí, la discriminación debe ser siempre relativa al contexto de percepción, ${ }^{30}$ esto es, las restantes cosas que el sujeto puede percibir en un momento particular en su entorno perceptual. Así, en este primer caso, la discriminación debe darse entre dos entidades de diferentes tipos situadas en dicho contexto en el mismo momento pero en diferentes coordenadas. El fundamento para la diferenciación estará

\footnotetext{
${ }^{30}$ Kelly 2001 también destaca el papel que el contexto de percepción debe desempeñar en la evaluación de propiedades perceptuales, aunque con otros fines. Sin embargo, coincidimos con él en señalar la necesidad de incorporar datos acerca de dicho contexto al analizar el contenido perceptual no conceptual.
} 
dado por un rasgo inherente a la representación no conceptual de cosa: la forma pura de la entidad en cuestión. Por este término no debe entenderse nada relacionado con la idea de forma aristotélica; no se habla aquí de forma en términos metafísicos sino en términos físicos. ${ }^{31}$ La forma pura de los objetos, por lo tanto, es la forma, el volumen y las tres dimensiones que percibe el sujeto cuando percibe el objeto sin aplicarle concepto sortal alguno; entre las características que supervienen de las cualidades de los puntos de percepción que conforman una cosa se halla el modo en el cual percibimos, en el nivel no conceptual, la forma física que posee la entidad causalmente responsable de dicha información perceptual. En el nivel conceptual más básico, ${ }^{32}$ esta forma de los objetos sirve al sujeto como evidencia para su clasificación bajo diferentes sortales; la correspondencia entre la forma del objeto y la forma comunitariamente asociada (Zerbudis 2007) a un determinado prototipo de objeto sortalizado permite la percepción de la entidad como un miembro de la clase de objetos que caen bajo el correspondiente sortal: así, lo que en este nivel conceptual nos permite percibir una taza qua taza es la forma del objeto ante nosotros, la similitud entre su forma y la forma canónica de taza. La forma física, tal y como es percibida en el nivel conceptual, es entendida como forma-de (en este caso, forma-de-taza). A diferencia de ésta, la forma pura corresponde al modo en que la forma física de la entidad es percibida en un nivel no conceptual, haciéndose aquí abstracción de toda relación entre dicha forma física y sus similitudes con formas canónicas asociadas a sortales. De modo que una cosa que en el plano no conceptual nos parece esférica y de cierto tamaño y color rojo (ésta sería su forma pura), en el plano conceptual se nos presenta como una entidad clasificable bajo el sortal "manzana" (esto es, tiene forma-de-manzana): la forma pura corresponde a la figura tridimensional que percibimos, mientras que la forma-de implica la clasificación del objeto, en función de esa forma física, bajo un concepto de sortal. Teniendo en cuenta esta distinción, en un caso como el considerado en (1) el individuo discrimina entre ambos objetos en cuanto que los percibe como cosas (articuladas, persistentes) con una forma pura diferente, y es capaz además de localizarlos en diferentes coordenadas del espacio, en virtud del vínculo informacional que mantiene con cada uno. Así, la idea de una forma pura es el

${ }^{31}$ Esto es, en términos de lo que en inglés llamaríamos "shape".

${ }^{32}$ En un nivel de este tipo podríamos hallar juicios perceptuales básicos, reportes de percepción como "El gato está sobre la cama" o "este lápiz es verde", o incluso juicios del tipo "Cholito está sobre la cama". 
equivalente no conceptual al concepto de sortal, del mismo modo en que la representación de cosa equivale, en el plano no conceptual, al concepto de objeto sortalizado en el conceptual.

(2) El segundo caso exige que sea posible discriminar entre dos objetos diferentes pero del mismo tipo; esta identidad de tipo nos impide valernos entonces de la forma pura, puesto que ésta puede ser idéntica en ambos objetos. Sin embargo, cuando ambos objetos son perceptibles en el mismo momento en el campo perceptual, es perfectamente posible, contrariamente a lo que sostiene Evans, diferenciarlos meramente por sus locaciones en el espacio egocéntrico, si previamente se los ha recortado como cosas, esto es, como posibles anclajes o fuentes de vínculos informacionales. Así, un organismo no conceptual puede discriminar entre un objeto $G$ situado a su derecha y otro $G$ situado a su izquierda, siempre que pueda percibirlos, a pesar de la idéntica forma pura, como dos cosas con la misma forma en dos lugares diferentes. El caso que presenta problemas serios es aquel donde los dos objetos aparecen en el contexto perceptual exactamente en el mismo punto del espacio egocéntrico, pero en dos momentos diferentes; el sujeto percibe un $G$ (una cosa con cierta forma pura) en el lugar $L$ en $t_{1}$, y, tras un lapso en el cual no recibe información del entorno (supongamos que cierra los ojos), percibe un G (una cosa con la misma forma pura) en $L$ en $t_{2}$. En este caso, la discriminación sólo podría lograrse si las formas puras de ambas entidades fueran ligeramente diferentes o si el punto espacial en que se encuentra el $\mathrm{G}$ en $t_{2}$ fuera mínimamente diferente, una locación $L^{*}$ (puesto que eso atentaría contra el criterio de minimización de cambios). ${ }^{33} \mathrm{De}$ excluirse estas posibilidades, la discriminación entre ambos objetos es imposible. Sin embargo, todo lo que se pide a la representación de $\cos a$ es que satisfaga el requisito de discriminación al menos con la misma eficacia con que lo hace el concepto de Objeto sortalizado. Un examen de los componentes del criterio de individuación ligado a un concepto de sortal muestra que éste sólo permite discriminar entre objetos del mismo tipo presentes al mismo tiempo, ${ }^{34}$ pero no

\footnotetext{
${ }^{33}$ Esto es conflictivo, porque supone una diferenciación entre cosas y organismos vivos (excluyendo a las plantas) en el nivel no conceptual: el criterio de minimización de cambios, aplicado a cosas, le permite suponer (no inferencialmente) al sujeto que el objeto no se moverá por sí mismo cuando deje de mirarlo, y que, por ende, conservará su lugar ceteris paribus. De un organismo, en cambio, se prevé que pueda moverse. Creemos que no es imposible sostener que esta discriminación pueda darse en seres no conceptuales tomando como base los estudios que Karmiloff-Smith ha realizado sobre la percepción en los bebés. Véase Karmiloff-Smith 1994.

${ }^{34}$ Es por eso que permite contar objetos del mismo tipo.
} 
diferencia un objeto de otro del mismo tipo en casos de sustitución del objeto; aun si apeláramos a la identificación de los objetos, ésta resultaría insuficiente para estos casos. Así que, debido a los criterios de minimización de cambios, el sujeto irremediablemente considerará el segundo objeto idéntico al primero, sea que perciba las entidades como cosas o como objetos sortalizados.

(3) El último caso es presentado por Evans mismo en el párrafo citado páginas atrás: se trata de dos objetos de diferente tipo (una estatua y un pedazo de arcilla) pero situados en las mismas coordenadas espacio-temporales. El caso impide que la discriminación se realice apelando a la localización del objeto percibido como cosa, y tampoco es posible apelar a la forma pura de las entidades: ambos objetos, a pesar de ser de diferente tipo, tienen la misma forma pura. ${ }^{35}$ En este caso, la discriminación sólo puede hacerse mediante el concepto de atención: $:^{36}$ así, si el sujeto tiene interés en rastrear la entidad "pedazo de arcilla", seleccionará del entorno o escenario una protoproposición diferente de la que seleccionaría otro sujeto que deseara rastrear la entidad "estatua"; si bien ambos recortarían los mismos conjuntos de puntos del escenario en ese momento, el rastreo de las cosas a las que ambos se referirían con "esto" mostrarían caminos divergentes a lo largo del tiempo. De hecho, un mismo sujeto puede prestar atención a ambas cosas al mismo tiempo, almacenando después, como información relevante del entorno, protoproposiciones diferentes del tipo [la arcilla es roja] y [la estatua es enorme]. Este recorte diferente permitirá que más tarde el sujeto demuestre ser capaz de reconocer una cosa como la misma estatua (aun cuando toda la arcilla original hubiera sido reemplazada por otra), ${ }^{37}$ y otra cosa como la misma arcilla (que ahora deja de tener la forma que tenía)..$^{38} \mathrm{El}$ concepto de atención cumple aquí, entonces, el mismo papel que el de los

35 Tampoco es posible apelar a la distinción entre la relación de constitución y la de identidad, mientras sea posible rastrear tanto objetos que caen bajo sortales (estatuas) como objetos que caen bajo términos de masa (pedazos de arcilla). Recuérdese que no nos interesa la identidad del objeto, sino la posibilidad de discriminarlo de otros.

${ }^{36}$ Este concepto guarda cierta similitud con el concepto de capacidad recognicional en Evans ( $V R$, cap. 8).

${ }^{37}$ Dejamos de lado en este punto problemas relativos a si, en un caso como éste, la estatua seguiría siendo la misma estatua. Un lector interesado debería leer los debates en torno al Barco de Teseo. Un resumen de este debate puede encontrarse en Lowe 1983.

${ }^{38}$ Huelga decir que estos juicios de identidad no serán formulados, en el nivel de las protoproposiciones, mencionando que se trata de arcilla o estatuas, sino simplemente en protoproposiciones del tipo [esta cosa es la misma que la cosa roja de antes]. 
conceptos de estatua y arcilla: fija la atención del individuo y ancla su capacidad de rastreo en una dimensión de la forma pura que se tiene ante sí. ${ }^{39}$

La apelación a la representación de cosa permite entonces discriminar las diferentes entidades presentes en un determinado contexto de percepción al menos tan finamente como lo hace la identificación de las mismas a través de conceptos de sortal, permitiendo que el sujeto localice una entidad y ancle un vínculo informacional en un objeto en el campo perceptual (cosa que, como vimos, la capacidad de localización proporcionada por el vínculo informacional no puede hacer por sí misma y en forma aislada). Queda entonces por descartar la posibilidad de que esta representación de cosa sea en realidad un concepto básico.

\section{4. "Cosa": ¿concepto demostrativo o noción no conceptual?}

Es posible, entonces, construir una representación de cosa (o protoobjeto) de naturaleza no conceptual que forme parte de la información perceptual y pueda ser usada como herramienta de discriminación entre entidades, como correlato de la identidad conceptual de las mismas. Sin embargo, una objeción esperable proveniente del conceptualismo sostendría que dicha representación posee, en realidad, una naturaleza conceptual, si bien se trata de un concepto muy básico. En esta dirección podría argumentarse que, dado que conceptos como "cosa", "objeto", "entidad", cumplen en nuestras inferencias el papel de testaferros de sortal mencionados páginas atrás, deben existir, en consecuencia, conceptos de cosa, objeto o entidad; aunque existen razones que impiden considerarlos como conceptos sortales genuinos, nada impide entenderlos como conceptos. Ahora

${ }^{39} \mathrm{El}$ evaluador anónimo se pregunta en este punto si es posible que un sujeto rastree una entidad de tipo $\mathrm{S}$ sin poseer un concepto $S$ correspondiente. Creemos que esto es posible, siempre y cuando se considere que el sujeto no está prestando atención o rastreando la entidad $\mathrm{S}$ en cuanto objeto $S$, sino la entidad en cuanto cosa. Así, no es necesario sortalizar o conceptualizar un objeto (aplicarle un concepto que nos diga qué es) para rastrearlo o prestarle atención: es posible, por ejemplo, prestar atención a un matiz de color, a una sombra proyectada en la pared, a un aroma, y es posible asimismo recordar dichas propiedades para reencontrarlas en un futuro. Los animales, suponiendo que no cuenten con conceptos, hacen esto mismo: así, un gato puede prestar atención a una cosa que se mueve en su campo visual. En este trabajo sostenemos que las propiedades que anclan nuestra atención en el campo perceptual son las que conforman lo que hemos llamado "forma pura", en oposición a la percepción de una forma correspondiente a un objeto según pueda ser éste conceptualizado. Lo que podemos rastrear y aquello a lo que podemos prestar atención sin valernos para ello de conceptos es, entonces, esta "forma pura". 
bien, un concepto tal no se halla asociado a criterio de identidad alguno, y por lo tanto sólo puede tratarse de un concepto más básico que los conceptos sortales habituales.

Un modelo posible para concebir este concepto sería el de concepto demostrativo, del que McDowell (2003) se vale para hacer frente al problema que el grano fino del contenido perceptual presenta al conceptualismo: un concepto de este tipo debería ser lo suficientemente fino como para poder captar todos los matices de las propiedades perceptuales, cosa que puede realizarse uniendo simplemente el concepto genérico que las cubre a un demostrativo ("este color", "esta textura"). Podríamos pensar, entonces, que ante cada conglomerado articulado y persistente de puntos presentes en el entorno perceptual, ante cada forma pura, el sujeto aplica un concepto demostrativo del tipo "esta cosa". Si esto es así, nuestra propuesta carecería de sentido, puesto que lo que buscamos es proporcionar correlatos no conceptuales para los conceptos de sortal que permiten la discriminación de objetos-fuente en el contenido perceptual. Por supuesto, de hecho existen conceptos de este tipo, conceptos obtenidos tal vez a partir de los conceptos de los objetos particulares y la abstracción de todas sus particularidades; la postulación de un concepto de "cosa" en el nivel conceptual no implica necesariamente la inexistencia de una representación no conceptual de cosa. El punto en discusión con el conceptualista no es la existencia o inexistencia de un concepto de este tipo, sino si el contenido perceptual requiere necesariamente la aplicación de un concepto de este tipo, si seres no conceptuales pueden percibir las entidades como cosas aun careciendo del concepto básico de "cosa". Un primer punto a favor de la consideración de la existencia de una noción no conceptual de cosa son los elementos de prueba conductuales que muestran que los seres no conceptuales tratan a diferentes sectores de su entorno perceptual como se trata a una cosa, a un objeto material: esquivan los objetos a su paso, los muerden, los tocan, etc. Por lo tanto, a menos que se quiera hacer de los animales, por ejemplo, seres conceptuales — aun cuando sólo se les atribuyan conceptos muy básicos-, ha de admitirse que existe algo así como una representación no conceptual de cosa diferente del concepto básico de cosa. ${ }^{40}$ Un argumento de este tipo es presentado por Bermúdez: ${ }^{41}$ que la conducta de bebés y animales se pueda

\footnotetext{
${ }^{40}$ Desde luego, es evidente que el supuesto operante bajo todo este trabajo es discontinuista: existe un salto cualitativo entre seres conceptuales y no conceptuales, y la idea de que los segundos cuenten con conceptos, aun cuando sean muy básicos, debe ser evitada.

${ }^{41}$ Véase Bermúdez 1995, pp. 189 y ss.
} 
describir como si fuera resultado de la aplicación de conceptos (en particular, del concepto de objeto) no implica necesariamente que estos seres posean conceptos; puede tratarse de un registro de la presencia de objetos-fuente en términos meramente prácticos y no inferenciales o conceptuales.

Este tipo de argumento muestra con claridad la relación existente entre las representaciones no conceptuales ("cosa", pero también "aquí", "arriba", etc.) y la acción del sujeto. La prueba de que existe una representación de cosa radica en las acciones que el individuo está dispuesto a realizar (y de hecho realiza) ante ciertas porciones de su entorno perceptual, del mismo modo en que la prueba de que existen nociones como arriba y al lado radica en las conductas diferenciadas que los individuos llevan adelante ante entidades situadas en diferentes puntos de dicho entorno. Sin embargo, este argumento no basta por sí solo. En este sentido, el punto fuerte a favor del conceptualista es que, en debates de este tipo, el modo en que entendamos la naturaleza de ciertos contenidos mentales parece ser una cuestión de preferencias personales: los argumentos a favor y en contra de la naturaleza conceptual de conceptos muy básicos no parecen ser nunca concluyentes. Aun así, es posible apelar a las características que los autores de uno y otro bando consideran que poseen los contenidos no conceptuales, e indagar si son poseídos por la representación de $\cos a$.

Hay muchas teorías acerca de las características que un contenido debe poseer para ser considerado contenido conceptual; ${ }^{42}$ en este trabajo retomaré solamente las señaladas por Gunther (2003): ${ }^{43}$ de éstas, yendo por su negativa a las características del contenido no conceptual, sólo nos detendremos en una, la que podría presentar, más que las demás, ${ }^{44}$ problemas para la noción de cosa: la determinación de la

${ }^{42}$ Véase la compilación de Gunther 2003a.

${ }^{43}$ Se trata de composicionalidad, relevancia cognitiva, determinabilidad de la referencia y independencia de la fuerza.

${ }^{44}$ La composicionalidad, creemos, puede darse tanto en el plano conceptual como en el no conceptual, mientras que podría sostenerse que las protoproposiciones respetan el Principio de Generalidad de Evans y, aun así, sus componentes son claramente no conceptuales. La independencia de la fuerza, por su parte, no es un rasgo del contenido no conceptual que implique la noción de cosa: las protoproposiciones conformadas por ésta y el recorte de una de sus propiedades conforman el contenido de un estado mental que no puede asumir otra fuerza que no sea la protoasertórica, por así decirlo. Por último, ilusiones ópticas como espejismos y hologramas son percibidos por todo individuo como cosas en el entorno perceptual, sin que esto modifique su creencia de que no hay nada en el lugar donde las supuestas entidades se localizan; así que la relevancia cognitiva tampoco se cumple. 
referencia. Suponiendo que el uso del demostrativo "esto" 45 tiene por sentido un modo no conceptual de presentación del objeto referente, esto es, la percepción de éste en el nivel no conceptual como una cosa, dicha noción sería entendida como no conceptual si el individuo no pudiera determinar la referencia de "esto", es decir, si no pudiera conocer la esencia del objeto referente, si no pudiera identificarlo o localizarlo, o si no pudiera reconocerlo o reidentificarlo. Dada nuestra noción de $\cos a$, resulta evidente que el individuo no necesita ser capaz de identificar el objeto (lo que es equivalente, en algunas lecturas, a conocer su esencia); ${ }^{46}$ si bien en muchos casos la clasificación o sortalización se realiza automáticamente, en casos de ignorancia de sortal o en los que el individuo es un organismo no conceptual, la identificación no es posible: solamente lo es la capacidad de discriminar el referente de otros objetos en el contexto perceptual. Así, al menos en dos sentidos de "determinación de la referencia", el contenido de la noción de $\cos a$ no requiere que el sujeto que percibe determine la referencia. Puede alegarse que el individuo sí la determina en otro sentido, en cuanto que es capaz de localizar al objeto referente. Pero dicha localización podría suceder sólo en el espacio egocéntrico y no en el objetivo (el individuo podría hallarse perdido y aun así saber que delante de él hay una cosa) y, por lo tanto, la localización de las cosas es, en primera instancia, puramente no conceptual. Sumemos a esto que el requisito de discriminación aparece porque Evans juzga que la localización, por sí sola, no es capaz de discriminar el objeto en algunos casos. Por último, podría alegarse que el individuo puede determinar que "esto" refiere a un determinado objeto en cuanto puede reconocerlo. Sin embargo, es posible que la entidad se nos presente como una cosa y que seamos incapaces de reconocerla más adelante, cuando se nos presenta nuevamente; de hecho, esto suele suceder cuando no prestamos atención al objeto, esto es, cuando no lo almacenamos en la memoria como parte de información relevante sobre el entorno. Si esto es así, entonces el individuo puede percibir una entidad en forma no conceptual, como una cosa en el contexto conceptual, y tener un estado mental que implique a la entidad sin

${ }^{45}$ Cabe señalar que no hay una correspondencia impecable entre el uso de demostrativos y modos de presentación demostrativos del objeto referente: es posible referirse a un objeto mediante un demostrativo aun cuando el modo de presentación de aquél no sea demostrativo; véase el caso del uso de "esta manzana" para referirse a una manzana que, por estar en un laberinto de espejos, el sujeto que percibe no puede localizar.

${ }^{46}$ En aquellas que sostienen que la propiedad de caer bajo un determinado sortal constituye la esencia del objeto. Pero véase Mackie 1994. 
por ello ser capaz de determinar de qué tipo de cosa se trata, en qué parte del mundo se halla localizada, o sin ser capaz de reconocerla. ${ }^{47}$

Por lo tanto, la noción de cosa posee todos los rasgos propios de los contenidos no conceptuales. Ahora bien, estos rasgos se dan en la noción de cosa en términos débiles: si bien el contenido de la representación de cosa podría no permitir al sujeto que percibe inferir creencias, ni determinar al referente, podría permitirlo perfectamente también. Tal vez por ello es tentador negar la existencia de una representación de cosa operante en la percepción tanto de humanos como de seres no conceptuales para señalar que "cosa" sólo puede consistir en un concepto. Sin embargo, para los fines presentes, no es necesario que la noción cumpla con estos rasgos en un sentido fuerte (esto es, que los cumpla siempre). Basta que exista la posibilidad de que, en ciertas ocasiones, el individuo pueda tener el contenido aun cuando estos rasgos propios de la conceptualidad no se apliquen. El hecho de que se apliquen en múltiples ocasiones se puede explicar por el salto inmediato que el ser humano (en cuanto organismo conceptual) da de la percepción de algo como una cosa a la clasificación de ese algo como un Objeto sortalizado y, por lo tanto, como una entidad clasificable bajo el concepto de "objeto". De modo que es posible sostener, en estos términos, que la representación de cosa es algo diferente del concepto de cosa, y que su naturaleza es enteramente no conceptual, aun cuando se halla estrechamente ligado, en seres conceptuales, a la posibilidad de aplicación de dicho concepto en la experiencia.

\section{Conclusión: contenido no conceptual y cosas}

Al considerar las ideas de Evans referentes al pensamiento singular y a las Ideas fundamentales de objeto que aparecen en él advertimos una característica común a diversos planteamientos sobre el contenido no conceptual de la percepción: a fin de que sea posible caracterizar este contenido como no conceptual, los autores se han esforzado por

\footnotetext{
${ }^{47}$ Con esto se resuelve la tensión que genera este criterio de Gunther para el tratamiento de los sentidos de los demostrativos: si los demostrativos tienen contenidos no conceptuales, se sigue del criterio de Gunther que es imposible determinar su referencia (agradezco a Eleonora Orlando haberme señalado este punto). Sin embargo, si se considera que basta la posibilidad de discriminar el objeto de otros para afirmar que se ha determinado la referencia (aunque sea mínimamente), entonces el criterio de Gunther no sólo no presenta problemas para sostener que las cosas pertenecen al nivel no conceptual, sino que además se tratará de un criterio demasiado estricto para los sentidos no conceptuales de los demostrativos. Hay más formas de determinar la referencia que las que Gunther sugiere.
} 
vincular sujeto y objeto en términos que no requieran la aplicación de conceptos por parte del primero para percibir el segundo. El sujeto ancla sus pensamientos en el objeto-fuente sin conceptualizarlo, sino sólo en virtud de una relación entre ambos de tal naturaleza que permite que el primero localice al segundo en el espacio. Ahora bien, en los planteamientos de este tipo, la localización por sí misma resulta insuficiente para ligar el sujeto al objeto: para ello es necesario percibir al objeto como tal, como algo más que una mera colección de qualias en el contexto de percepción. La localización resulta también insuficiente, por sí sola, para diferenciar diferentes objetos entre sí, especialmente cuando éstos se hallan localizados en las mismas coordenadas espacio-temporales. Así, un segundo elemento necesario para el contenido de la información perceptual, casi nunca explícitamente mencionado pero siempre implícitamente presente, es la discriminación del objeto, la percepción de una serie de puntos en el espacio como un objeto de cierto tipo, cuyas condiciones de identidad, adecuadas al tipo de entidad, permiten establecer sus límites y diferenciarlos de otros objetos. Localización e identificación son entonces las bases sobre las que las teorías no conceptualistas asientan el contenido perceptual.

No obstante, como hemos visto, la identificación implica la aplicación de conceptos sortales y, en consecuencia, tiene su lugar en el plano del pensamiento conceptual. Es necesario entonces dar con algo que cumpla, en el nivel no conceptual, el papel que los conceptos sortales cumplen en el nivel conceptual y que la mera localización no puede proporcionar. Para ello nos hemos propuesto una serie de pasos: en primer lugar, desbrozar lo que el contenido perceptual necesita, de modo de ver que todo lo que se exige en realidad es la satisfacción del requisito de discriminación (esto es, la posibilidad de diferenciar objetos diferentes aun cuando estén en el mismo lugar). Este requisito puede ser satisfecho por una representación no conceptual de cosa: la cosa es construida a partir de puntos cualitativamente cargados en el escenario perceptual más básico, cuya homogeneidad cualitativa, una vez cumplidos ciertos requisitos, conduce a la percepción de los mismos como partes de un todo articulado, persistente y con una cierta forma. Esta representación, aunque guarda relaciones con el concepto de "cosa", no puede ser identificada con él, y tiene el mismo poder discriminativo, en el contexto perceptual, que la de la consideración de Objetos sortalizados en el plano del pensamiento.

Si esto es así, el contenido no conceptual se apoya tanto en la localización como en la discriminación de cosas, sin necesidad de 
la intervención de conceptos. El individuo se vincula a los objetos en su entorno cuando puede localizarlos como cosas en su espacio egocéntrico, haciéndolos términos o anclajes de sus vínculos informacionales con el entorno. De este modo, el contenido informacional de la percepción corresponde completamente a su correlato conceptual en el nivel del pensamiento, pero depende enteramente de habilidades y capacidades básicas del sujeto. ${ }^{48}$

\section{BIBLIOGRAFÍA}

Bermúdez, J.L., 1995, “Nonconceptual Content: From Perceptual Experience to Subpersonal Computacional States", en Gunther 2003a, pp. 183216.

, 1994, "Peacocke's Argument against the Autonomy of Nonconceptual Representational Content", en Gunther 2003a, pp. 293-308.

Brandom, R., 1994 (2005), Hacerlo explícito. Razonamiento, representación y compromiso discursivo, Herder, Barcelona.

Dretske, F., 1981, "Sensation and Perception", en Gunther 2003a, pp. 2542.

Evans, G., 1982a, The Varieties of Reference, Clarendon Press, Oxford.

__ 1982b (1996), "La comprensión de los demostrativos", Ensayos filosóficos, trad. Alejandro Tomasini Bassols, Instituto de Investigaciones Filosóficas-UnAM, México, pp. 319-352.

Faria, P., 2001, "Discriminaçao e Conhecimento Direto", en G. Hurtado (comp.), Subjetividad, representación y realidad, Benemérita Universidad Autónoma de Puebla/Facultad de Filosofía y Letras-Unam, Puebla, pp. 9-29.

Geach, P.T., 1967, "Identity", Review of Metaphysics, vol. 21, pp. 3-12.

- 1962, Reference and Generality: an Examination of some Medieval and Modern Theories, Cornell University Press, Ithaca.

Griffin, N., 1977, Relative Identity, Clarendon Press, Oxford.

Gunther, Y.H., 2003a, Essays on Nonconceptual Content, MIT Press, Cambridge, Mass.

— , 2003b, "General Introduction", en Gunther 2003a, pp. 1-20.

Hirsch, E., 1982, The Concept of Identity, Oxford University Press, Nueva York.

Karmiloff-Smith, A., 1994, Más allá de la modularidad, Alianza, Madrid.

Kelly, S., 2001, "The Nonconceptual Content of Perceptual Experience: Situation Dependence and Fineness of Grain", en Gunther 2003a, pp. 223230.

${ }^{48}$ Agradezco, por sus comentarios a diferentes versiones de este trabajo, a los evaluadores anónimos de Crítica, a Eleonora Orlando, a Ezequiel Zerbudis y a Laura Skerk. 
Lowe, E.J., 1983, "On the Identity of Artifacts", The Journal of Philosophy, vol. 80 , no. 4 , pp. 220-232.

Mackie, P., 1994, "Sortal Concepts and Essential Properties", The Philosophical Quaterly, vol. 44, no. 176, pp. 311-333.

McDowell, J., 2005, "Evan's Frege", en J.L. Bermúdez (comp.), Thought, Reference and Experience. Themes from the Philosophy of Gareth Evans, Oxford University Press, Oxford, pp. 42-65.

— 2003 , "Nonconceptual Content”, en Gunther 2003a, pp. 75-90. Mass. 1996, Mind and World, Harvard University Press, Cambridge,

—, 1990 , "Peacocke and Evans on Demonstrative Content", Mind. New Series, vol. 99, no. 394, pp. 255-266.

Peacocke, C., 2002, "Postscript: the Relations between Conceptual and Nonconceptual Content", en Gunther 2003a, pp. 318-322.

- 2001, "Does Perception Have a Nonconceptual Content?", The Journal of Philosophy, vol. 98, pp. 239-264.

- , 1994, "Nonconceptual Content: Kinds, Rationales and Relations", en Gunther 2003a, pp. 309-322.

- 1992, "Scenarios, Concepts and Perception", en Gunther 2003a, pp. 107-132.

- 1991, "Demonstrative Content: a Reply to John McDowell”, Mind. New Series, vol. 100, no. 1, pp. 123-133.

- 1983, Sense and Content, Oxford University Press, Oxford.

Russell, B., 1910, "Conocimiento directo y conocimiento por descripción", Misticismo y lógica, trad. José Rovira Armengol, Edhasa, Barcelona, 1987, pp. 209-230.

Sainsbury. R.M., 1985, "Critical Notice: The Varieties of Reference by Gareth Evans", Mind. New Series, vol. 94, no. 373, pp. 120-142.

Strawson, P.F, 1959, Individuos, trad. Alfonso García Suárez y Luis M. Valdés Villanueva, Taurus, Madrid, 1989.

Thomson, J.J., 1998, "The Statue and the Clay", Noûs, vol. 32, no. 2, pp. 149-173.

Wiggins, D., 2001, Sameness and Substance Renewed, Harvard University Press, Cambridge, Mass.

Zerbudis, E., 2007, "Términos clasificatorios, esencias e identidad", en P. Brunsteins y A. Testa (comps.), Conocimiento, normatividad y acción, Facultad de Filosofía y Humanidades de Córdoba, Córdoba, pp. 519-524.

Recibido el 31 de julio de 2007; revisado el 24 de abril de 2009; aceptado el 13 de mayo de 2009. 